\title{
ANÁLISE DA QUALIDADE DOS SERVIÇOS PRESTADOS POR UM ESCRITÓRIO DE CONTABILIDADE NA CIDADE DE FRANCA-SP PELA FERRAMENTA SERVPERF: ESTUDO DE CASO
}

\author{
Ana Paula Taveira Alves (Centro Universitário Municipal de Franca) \\ anapaulataveira5@gmail.com \\ Paulo Renato Pakes (Universidade Federal de São Carlos) paulopakes@gmail.com \\ Adriana Figueiredo de Morais Abreu (Universidade de Franca) nanafigueredo17@gmail.com
}

\section{Resumo}

$\mathrm{Na}$ atualidade, a qualidade do serviço passou a ser um quesito de extrema importância, visto que é por meio dela que as empresas conseguem satisfazer os clientes. Assim, adequar a qualidade esperada à qualidade percebida desses serviços se tornou um desafio. Uma das ferramentas de medição conhecida mundialmente é o modelo SERVPERF que é utilizado para medir e consequentemente avaliar a qualidade do serviço mediante a percepção dos clientes. Assim sendo, este trabalho teve como objetivo medir a qualidade do serviço prestado em um Escritório de Contabilidade da cidade de Franca no estado de São Paulo. Para isso, adaptou-se o modelo SERVPERF na perspectiva de avaliar a satisfação dos serviços prestados, de acordo com a concepção dos seus clientes, no intuito de identificar os pontos fortes e fracos, bem como propor melhorias para a organização. Para esse trabalho, o método de pesquisa utilizado foi o estudo de caso, por meio da aplicação de um questionário que foi encaminhado para uma amostra de respondentes, composta por pessoas chaves dos clientes de diversos seguimentos e atividades, aleatoriamente. Constatou-se como principais resultados que a empresa $\mathrm{ABC}$ apresenta qualidade em serviços perante o que é constatado pela maioria dos respondentes da pesquisa, entretanto, foram sugeridas melhorias para alguns atributos como a falta de visita na empresa cliente, à estrutura física e os equipamentos utilizados, que apresentaram baixa satisfação dos clientes.

\section{Palavras-Chaves: Qualidade; Serviços; Contábeis; SERVPERF.}

\begin{abstract}
At present, the quality of the service has become a matter of extreme importance, since it is through this that the companies can satisfy the customers. Thus, adjusting the expected quality to the perceived quality of these services has become a challenge. One of the measurement tools known worldwide is the SERVPERF model that is used to measure and consequently evaluate the quality of the service through the perception of the customers. Therefore, this study had as objective to measure the quality of the service provided in an Accounting Office of the city of Franca in the state of São Paulo. For this, the SERVPERF model was adapted to evaluate the satisfaction of the services provided, according to the design of its clients, in order to identify the strengths and weaknesses, as well as propose improvements for the organization. For this study, the research method used was the case study, by means of the application of a questionnaire that was sent to a sample of respondents, composed of key people of the clients of several segments and activities, at random. It was verified as main results that the company $\mathrm{ABC}$ presents quality in services before what is verified by the majority of the respondents of the research, however, some improvements were suggested for some attributes like the lack of visit in the client company, the physical structure and the equipment used, which presented low customer satisfaction.
\end{abstract}

Keywords: Quality; Services; Accounting; SERVPERF. 


\section{Introdução}

Em tempos de dificuldades financeiras e com a concorrência cada vez mais acirrada, as empresas estão sendo obrigadas a adotarem estratégias e práticas eficazes no mercado de negócios que propiciem um meio mais competitivo.

Para alcançar um serviço de qualidade e atender as necessidades dos clientes é essencial melhorar os processos e os serviços prestados através de uma gestão da qualidade eficiente.

Compete a cada escritório contábil compreender sua capacidade de serviço e com qual qualidade este vai chegar até o cliente, tornando-se provável atingir de forma progressiva bons resultados, através da melhoria contínua, visto que, com a satisfação dos clientes torna-se possível a recomendação de novos clientes e assim consecutivamente.

Mediante o contexto, o presente estudo tem por objetivo medir a qualidade do serviço prestado de consultoria e assessoria realizada por um escritório contábil na percepção de seus clientes, utilizando como base o modelo SERVPERF.

\section{Referencial teórico}

\subsection{Serviços}

A sociedade atual busca cada vez mais consumir não só os produtos, mas também experiências que garantem prazer e bem-estar. Com isto, a esfera de serviços vem ganhando importância na economia mundial e sendo considerada uma ótima oportunidade de diferencial competitivo. $\mathrm{Na}$ visão de Grönroos (1995), um serviço é um processo, consistindo em uma série de atividades mais ou menos intangíveis que, podem, ocorrerem nas interações entre o cliente e os funcionários de serviços e/ou bens físicos e/ou sistemas do fornecimento de serviços e que são fornecidas como soluções para problemas do cliente.

Para Lovelock e Wrigth (2001), serviço é assimilado como um ato ou comportamento basicamente intangível, que produz benefícios para os clientes por meio de uma mudança desejada no recebedor do serviço. Ainda, para Lovelock et al (2011) os serviços são definidos como ações econômicas em que uma parte oferece algo à outra.

\subsection{Qualidade}


De acordo com Oliveira et al. (2006), as empresas precisam produzir seus produtos e serviços com qualidade, não apenas como uma estratégia de distinção no mercado, mas como uma condição de existência.

O termo qualidade possui diversos significados. Para Andretti (2006), significa um conceito subjetivo que está diretamente relacionado às percepções de cada pessoa. Ainda, para Fischer et al. (2009), qualidade é o nível em que um grupo de característicos específicos atende a exigências, salientando ainda que os quesitos de qualidade são descritos pelos clientes ou pela sociedade, em forma de esperança e desejos.

A gestão da qualidade total, mais conhecida como Total quality management (TQM), é definida segundo Toledo et al. (2014), como uma abordagem e filosofia de administração integrada que apresenta um conjunto de práticas destacando a melhoria contínua, atendimento das expectativas e necessidades dos clientes, como foco a redução do retrabalho, trabalho em equipe, relacionamento próximo dos fornecedores, clientes e outros enfoques.

\subsection{Serviços contábeis}

De acordo com o Conselho Federal de Contabilidade (CFC, 2018), a contabilidade é uma ciência social que tem como objeto de trabalho o patrimônio da empresa. As principais dimensões da qualidade que devem estar presentes em serviços contábeis são a confiabilidade, relevância, tempestividade, compreensibilidade e comparabilidade. A confiabilidade pode ser considerada quando uma informação não apresenta erros importantes, conseqüentemente os clientes tenham confiança no serviço. Já a relevância, compara-se com a importância que aquela notícia possui para o consumidor e sua tomada de decisão. A tempestividade está ligada ao tempo, dado que uma notícia deve chegar ao cliente em tempo hábil e sendo os prazos alterados, devem ser informadas as razões. A compreensibilidade refere-se ao fato das notícias, devem possuir utilidade e serem totalmente compreendidas pelos consumidores. Por fim, a comparabilidade compara-se com o fato dos clientes compararem as demonstrações contábeis da empresa ao longo do tempo e de seus concorrentes a fim de avaliar sua posição financeira e detectar possíveis falhas.

A principal função dos serviços contábeis é: registrar, organizar, demonstrar, analisar e acompanhar as modificações do patrimônio em virtude da atividade econômica ou social que a sociedade exerce no contexto econômico. Sua finalidade básica é o acompanhamento das atividades realizadas pela sociedade, no sentido indispensável de controlar o comportamento 
de seus patrimônios, na função elaboração e comparação dos resultados obtidos entre períodos analisados.

\subsection{Modelo SERVPERF}

É um modelo para mensuração da qualidade de serviços, o qual foi desenvolvido por Cronin e Taylor (1992) com relação ao modelo SERVQUAL, cujo diferencial, é fundamentado apenas na percepção de desempenho dos serviços. O modelo SERVPERF foi elaborado baseando no entendimento do desempenho dos serviços, visto que o mesmo é um instrumento de maior confiabilidade para mensurar a qualidade em prestação de serviços (CRONIN; TAYLOR, 1992).

O SERVPERF é constituído por 22 itens sobre o desempenho do serviço, que representa as cinco dimensões de qualidade desenvolvidas por Parasuraman, Zeithaml e Berry (1988). Os itens são analisados na escala Likert, na qual varia de 1 a 7 , sendo 1 discordo completamente e 7 concordo completamente e ainda, não possuem a análise dos gaps no complemento da pesquisa.

Afirmado por Cronin e Taylor (1992) que a qualidade percebida dos serviços antecede à satisfação do cliente, sendo essa afetada de maneira negativa aos propósitos de aquisição dos clientes. Ainda, os autores comentam que a qualidade dos serviços influencia mais na satisfação do cliente do que nas intenções de compra, isto é, o desempenho apresentado pela prestação de serviço gera satisfação e é o que realmente importa para o cliente.

Quadro 2: Instrumento SERVPERF 


\begin{tabular}{|c|l|}
\hline ITEM & \multicolumn{1}{|c|}{ IMPORTÂNCIA } \\
\hline 1 & Equipamentos modernos. \\
\hline 2 & Instalações fisicas são visualmente atrativas. \\
\hline 3 & Os empregados são bem-vestidos e asseados. \\
\hline 4 & A aparência das instalações físicas é conservada de acordo com o serviço oferecido. \\
\hline 5 & Quando algo é prometido em um certo tempo é cumprido. \\
\hline 6 & Quando há algum problema com a empresa, ela é solidária e o deixa seguro. \\
\hline 7 & Tem confiabilidade. \\
\hline 8 & Fornece o serviço no tempo prometido. \\
\hline 9 & Mantém seus registros de forma correta. \\
\hline 10 & Informa exatamente quando os serviçcos serão executados. \\
\hline 11 & Recebe serviço imediato. \\
\hline 12 & Empregados estão sempre dispostos a ajudar os clientes. \\
\hline 13 & Empregados não estão sempre ocupados para responder aos pedidos dos clientes. \\
\hline 14 & Empregados são confiáveis. \\
\hline 15 & O sentimento de se sentir seguro em negociar com os empregados da empresa. \\
\hline 16 & Empregados são educados. \\
\hline 17 & Suporte adequado da empresa para cumprir suas tarefas corretamente. \\
\hline 18 & Atenção individual. \\
\hline 19 & Empregados dão a você atenção pessoal. \\
\hline 20 & Empregados sabem das suas necessidades. \\
\hline 21 & Os objetivos da empresa são os seus objetivos. \\
\hline 22 & Horário de trabalho conveniente. \\
\hline
\end{tabular}

Fonte: Questionário de Importância dos itens (CRONIN; TAYLOR, 1992).

Sendo assim, o modelo SERVPERF emprega somente informações de desempenho de serviços, visto que os clientes entrevistados expressam suas especificações espontaneamente, comparando as percepções de desempenho com as expectativas de desempenho. Posto isso, a ferramenta SERVPERF confirma que medir diretamente as expectativas de desempenho não é necessário (CARRILLAT, et al., 2007).

\section{Método}

O presente estudo, no que diz respeito à finalidade, consiste em pesquisa aplicada, pois o tipo de contribuição visa produzir um conhecimento que possa ser aplicado na vida real, ajudando a alterar uma situação. Esse tipo de pesquisa busca propor alternativas que ajudem a melhorar ou transformar determinado aspecto do objeto de estudo. 
Sendo o procedimento dessa pesquisa do tipo descritiva, o trabalho identifica a satisfação dos clientes com a qualidade dos serviços prestados por um escritório de contabilidade. Visto que as propriedades importantes na avaliação é apontar os pontos fortes e fracos no serviço prestado.

Este trabalho se caracteriza como sendo um estudo de caso, uma vez que procura examinar de forma clara, a qualidade do serviço prestado de um escritório de contabilidade. Segundo Yin (2015), o estudo de caso é uma investigação prática que possui por objetivo, investigar um acontecimento.

Nesse contexto explicitado, o estudo de caso foi realizado dentro do escritório contábil, buscando conhecer a empresa e reunir informações detalhadas sistematicamente sobre um assunto, quais os pontos que estavam com mais dificuldades e quais as questões que gostariam de saber de seus clientes para poder alcançar uma melhoria contínua na organização. Assim, constata-se a importância na percepção de seus clientes do grau de satisfação com a qualidade dos serviços que lhes eram prestados pelos colaboradores da empresa.

Assim, para poder medir essa satisfação, ainda foi utilizado o método de pesquisa para coleta e análise dos dados, denominado por survey ou levantamento. Para Martins et al (2014), um survey caracteriza-se por objetivos claros, questões bem formuladas, planejamento adequado da coleta de dados, seleção de uma amostra representativa, instrumentos de coleta de dados confiáveis e válidos, análise apropriada dos dados, por meio de técnicas estatísticas. O levantamento sobre a pesquisa de opinião dos clientes do escritório contábil foi realizado por meio de um questionário eletrônico, construído com o uso da ferramenta Google Formulários, onde nada mais é do que a tradução dos objetivos específicos da pesquisa em perguntas sobre o problema proposto.

O questionário foi constituído, a partir de uma adaptação do modelo SERVPERF, para melhor compreensão dos entrevistados. Foi elaborado com 26 afirmações relacionadas à percepção dos serviços prestados pelos profissionais do escritório contábil pesquisado e 4 questões complementares relacionadas ao perfil do respondente. As afirmativas foram agrupadas em cinco dimensões da qualidade: confiabilidade, segurança, empatia, presteza e tangibilidade. Ainda, para cada afirmativa priorizou a escolha da escala Likert de sete pontos, devendo os respondentes indicar seu nível de concordância para mensurar o grau da satisfação: (1) discordo totalmente; (2) discordo em grande parte; (3) discordo em partes; (4) neutro; (5) concordo em partes; (6) concordo em grande parte e (7) concordo totalmente. 


\begin{tabular}{|c|c|c|}
\hline Dimensão & & Afirmativa \\
\hline \multirow{5}{*}{ Confiabilidade } & 1 & A empresa desempenha os serviços conforme prometido. \\
\hline & 2 & Os profissionais transmitem confiança aos clientes. \\
\hline & 3 & Os profissionais demonstram interesse em resolver os problemas quando surgem. \\
\hline & 4 & Os profissionais dão retorno sempre que prometem. \\
\hline & 5 & A empresa divulga comunicados (datas de vencimento, parcelamentos, eventos, etc) em tempo ideal. \\
\hline \multirow{3}{*}{ Segurança } & 6 & Você se sente seguro ao realizar transações com os profissionais da empresa. \\
\hline & 7 & Os profissionais da empresa são sempre respeitosos. \\
\hline & 8 & Os profissionais apresentam o conhecimento necessário para responder às dúvidas dos clientes. \\
\hline \multirow{5}{*}{ Empatia } & 9 & A empresa presta atendimento individual. \\
\hline & 10 & A empresa tem profissionais que lidam com os clientes de forma atenciosa. \\
\hline & 11 & Os profissionais da empresa entendem as necessidades de seus clientes. \\
\hline & 12 & A empresa visita os clientes suficientemente. \\
\hline & 13 & A empresa tem horários de expediente convenientes. \\
\hline \multirow{5}{*}{ Presteza } & 14 & A empresa mantem os clientes inteirados sobre o andamento dos trabalhos. \\
\hline & 15 & Nesta empresa, você recebe apoio e suporte suficientes dos funcionários. \\
\hline & 16 & Os profissionais da empresa estão sempre dispostos para responder as solicitações dos clientes. \\
\hline & 17 & Os profissionais são ágeis em fornecer informações por e-mail, Skype ou telefone. \\
\hline & 18 & A qualidade que se recebe é compatível com o preço que se paga. \\
\hline \multirow{6}{*}{ Tangíveis } & 19 & A empresa tem equipamentos modernos, adequados e confortáveis. \\
\hline & 20 & As instalações físicas da empresa são adequadas. \\
\hline & 21 & Os funcionários da empresa tem uma aparência profissional. \\
\hline & 22 & $\begin{array}{l}\text { A empresa apresenta os materiais associados com o serviço (relatórios, apresentações, folders, etc.) } \\
\text { com aparência adequada. }\end{array}$ \\
\hline & 23 & O sistema de envio e solicitação de documentação (Domínio Atendimento) é de fácil navegação. \\
\hline & 24 & $\begin{array}{l}\text { O sistema de envio e solicitação de documentação (Domínio Atendimento) permite a realização de } \\
\text { todos os serviços desejados. }\end{array}$ \\
\hline \multirow{3}{*}{ Complementar } & 25 & Eu recomendaria a empresa para outras pessoas. \\
\hline & 26 & $\begin{array}{l}\text { Eu consideraria a empresa minha primeira escolha ao contratar serviços de consultoria e assessoria } \\
\text { nessa área. }\end{array}$ \\
\hline & 27 & Qual seu tempo de experiência com a empresa. \\
\hline \multirow{3}{*}{$\begin{array}{l}\text { Perfil Do } \\
\text { Correspondente }\end{array}$} & & Sexo: Feminino ( ) Masculino ( ) \\
\hline & & Idade: \\
\hline & & Cargo do entrevistado: \\
\hline
\end{tabular}

Fonte: Autoria própria (2018).

Após o preenchimento dos questionários, as respostas foram submetidas a um processo de análises críticas. Nessa análise, destes 49 respondentes iniciaram o preenchimento do questionário e 2 abandonaram o questionário antes de finalizar.

Feita a análise dos questionários, concluiu-se com um total de 47 válidos, a verificação contínua, passando para o próximo processo que destaca seus resultados.

Diante o contexto, a pesquisa foi empregada de forma quantitativa em relação à abordagem do problema, em razão do instrumento estatístico na coleta de dados, ter sido um questionário, aplicando como modelo base a escala SERVPERF ajustada ao segmento de serviços contábeis. 
Foi realizado um levantamento bibliográfico, com o intuito de buscar conhecimentos mais aprofundados sobre os temas mais relevantes, elaborar o problema de pesquisa e estabelecer o objetivo central do estudo, através de uma análise qualitativa.

Assim para finalizar a pesquisa, se conduziu uma análise estatística descritiva dos resultados, intencionando identificar e validar os gaps encontrados. E após identificados, foram propostas melhorias para a organização.

\section{Resultados}

\subsection{A empresa}

A empresa $\mathrm{ABC}$ é uma organização de pequeno porte e prestadora de serviços contábeis que oferece serviços de assessoria, consultoria e auditoria nas áreas contábil, fiscal, tributária, trabalhista, societária e de gestão.

Situada no interior de São Paulo, na cidade de Franca, a empresa foi fundada em maio de 2006, possui mais de 12 anos de atuação no mercado, tendo como objetivo atender os mais diversos segmentos de atividades. O escritório atende 135 empresas clientes e aproximadamente 15 microempreendedores. No entanto, o foco desta pesquisa não abrangerá os microempreendedores por não existir uma frequência de atendimento.

Atualmente, a empresa $\mathrm{ABC}$ conta com 12 colaboradores especializados, seu horário de funcionamento é de segunda a sexta, funcionando 10 horas por dia, com início às $07 \mathrm{~h} 30 \mathrm{~min}$.

\subsection{Dados demográficos da amostra}

A população dessa pesquisa é composta por clientes do escritório contábil pesquisado, estes de todos os regimes tributários como: Simples Nacional, Lucro Presumido e Lucro Real. Em 2018, o escritório possui 135 empresas desses regimes citados que são feitas a contabilidade mensalmente. Essa amostra é classificada como amostragem por conveniência.

A amostra por conveniência envolve a pesquisa com indivíduos acessíveis ao pesquisador e reduz o tempo na coleta de dados (HAIR JUNIOR et al., 2005). Essa informação de quantidade de amostra e os e-mails desses clientes estão registrados no banco de dados da empresa pesquisada. Foi possível o acesso aos e-mails de todas as empresas e primeiramente optou-se por enviar o questionário às mesmas. No entanto, identificou-se erros em alguns e-mails, com isso, apenas 120 pessoas receberam o link para participar da pesquisa. Salienta-se que nem todas responderam o questionário, e com isso, a amostra obtida foi classificada como não 
probabilística por conveniência, concluindo com apenas 47 questionários válidos e 2 incompletos.

Todos os representantes da empresa receberam o convite para participar da pesquisa por e-mail, esse enviado para o correio eletrônico da empresa constando uma breve apresentação da pesquisa em andamento e um link com as perguntas do questionário. Essa etapa ocorreu no período de 15 de outubro de 2018 a 31 de outubro de 2018. Também foram enviados e-mails lembretes sobre o prazo de preenchimento e ressaltando a importância da avaliação para a pesquisa e para a empresa. Foi mantida em sigilo a identidade dos pesquisados, proporcionando maior liberdade para que esses expressassem suas opiniões. Para Oliveira (2012 p.83), nesses casos, é recomendável não solicitar que o informante assine ou coloque seu nome no instrumento de pesquisa. Ainda, para impedir o constrangimento da pessoa que vai responder um questionário é importante ser informado ás pessoas pesquisadas, que ninguém será identificado para poder preservar a identidade e garantir a validade e confiabilidade das informações que forem dadas.

Sendo assim serão apresentadas as características dos respondentes válidos citados anteriormente. Analisando, os resultados apresentados no geral indicam que na empresa avaliada houve muito mais satisfação do que insatisfação conforme mostrado nos dados da tabela 1 .

Tabela 1 - Dados totais do questionário 


\begin{tabular}{|c|c|c|c|c|c|c|c|c|}
\hline & $\begin{array}{c}\text { Totais } \\
\text { respondentes }\end{array}$ & $\begin{array}{c}\text { Concordo } \\
\text { totalmente }\end{array}$ & $\begin{array}{l}\text { Concordo em } \\
\text { grande parte }\end{array}$ & $\begin{array}{l}\text { Concordo } \\
\text { em partes }\end{array}$ & Neutro & $\begin{array}{c}\text { Discordo } \\
\text { em } \\
\text { partes }\end{array}$ & $\begin{array}{c}\text { Discordo } \\
\text { em grande } \\
\text { parte }\end{array}$ & $\begin{array}{c}\text { Discordo } \\
\text { Totalmente }\end{array}$ \\
\hline 1 & 48 & 33 & 12 & 2 & 0 & 1 & 0 & 0 \\
\hline 2 & 47 & 30 & 11 & 6 & 0 & 0 & 0 & 0 \\
\hline 3 & 48 & 31 & 13 & 3 & 0 & 1 & 0 & 0 \\
\hline 4 & 48 & 26 & 12 & 6 & 2 & 1 & 1 & 0 \\
\hline 5 & 48 & 32 & 11 & 3 & 0 & 1 & 0 & 1 \\
\hline 6 & 48 & 38 & 7 & 1 & 1 & 0 & 1 & 0 \\
\hline 7 & 48 & 43 & 4 & 1 & 0 & 0 & 0 & 0 \\
\hline 8 & 48 & 27 & 15 & 4 & 1 & 1 & 0 & 0 \\
\hline 9 & 48 & 35 & 11 & 2 & 0 & 0 & 0 & 0 \\
\hline 10 & 48 & 38 & 7 & 3 & 0 & 0 & 0 & 0 \\
\hline 11 & 48 & 31 & 15 & 1 & 1 & 0 & 0 & 0 \\
\hline 12 & 48 & 15 & 7 & 9 & 13 & 1 & 1 & 2 \\
\hline 13 & 48 & 35 & 9 & 2 & 1 & 0 & 0 & 1 \\
\hline 14 & 48 & 27 & 14 & 3 & 3 & 0 & 0 & 1 \\
\hline 15 & 48 & 33 & 11 & 4 & 0 & 0 & 0 & 0 \\
\hline 16 & 48 & 32 & 14 & 2 & 0 & 0 & 0 & 0 \\
\hline 17 & 48 & 26 & 13 & 8 & 0 & 0 & 1 & 0 \\
\hline 18 & 48 & 26 & 14 & 3 & 4 & 1 & 0 & 0 \\
\hline 19 & 48 & 35 & 7 & 1 & 5 & 0 & 0 & 0 \\
\hline 20 & 48 & 35 & 8 & 1 & 4 & 0 & 0 & 0 \\
\hline 21 & 48 & 37 & 9 & 1 & 1 & 0 & 0 & 0 \\
\hline 22 & 48 & 28 & 9 & 3 & 7 & 0 & 0 & 1 \\
\hline 23 & 48 & 35 & 5 & 6 & 2 & 0 & 0 & 0 \\
\hline 24 & 48 & 27 & 11 & 4 & 5 & 1 & 0 & 0 \\
\hline 25 & 48 & 41 & 6 & 0 & 0 & 0 & 0 & 1 \\
\hline 26 & 48 & 32 & 13 & 2 & 0 & 0 & 0 & 1 \\
\hline \multirow{2}{*}{ Total } & \multirow{2}{*}{1247} & 828 & 268 & 81 & 50 & 8 & 4 & 8 \\
\hline & & $66,40 \%$ & $21,49 \%$ & $6,50 \%$ & $4,01 \%$ & $0,64 \%$ & $0,32 \%$ & $0,64 \%$ \\
\hline
\end{tabular}

Fonte: Autoria própria (2018).

Diante dos dados apresentados, sugerem-se melhorias para sanar os gaps de desempenho, encontrados a partir da aplicação do SERVPERF adaptado. As ações de melhorias podem ser estruturadas através de planos de ações, determinando encarregados e prazos de implantação para cada ação recomendada, e em alguns casos medidas de ação preventiva.

Expõem-se adiante as melhorias necessárias de acordo com cada dimensão do questionário avaliada. As informações avaliadas como "discordo em partes", "discordo em grande parte" e "discordo totalmente" pelos respondentes e que tiveram uma porcentagem expressiva, foram avaliadas como pontos fracos, pois não alcançaram bom desempenho em relação às percepções dos clientes.

Na dimensão Confiabilidade, observa-se um grande nível satisfatório em relação à confiança. Porém, houve um percentual de pessoas insatisfeitas relacionadas ao retorno de informações no 
tempo estimado, no interesse dos profissionais em resolver os problemas quando surgem e o tempo ideal de divulgação dos comunicados. Para sanar esse índice negativo, uma observação direta seria a solução. Em relação aos profissionais não retornarem sempre que prometem e demonstrarem falta de interesse em resolver os problemas, teria que ser observado pelo supervisor de cada setor se os funcionários estão sempre de prontidão para atender os clientes e se existem outras tarefas ou distrações que impeçam a realização adequada do serviço. Sobre a divulgação dos comunicados em tempo ideal, seria necessário verificar se os e-mails e whatsapp dos clientes estão corretos, pois pode estar ocorrendo falhas na comunicação.

Na dimensão Segurança, os respondentes demonstraram ter confiança na empresa $\mathrm{ABC}$, notando-se que é organizada e regrada. Não há preocupação com essa categoria, pois a satisfação é notória.

Na dimensão Empatia, o nível de satisfação foi bem avaliado, o único fato identificado como ponto fraco foi de a empresa não visitar regularmente os clientes. Para tanto, foi sugerido montar um cronograma mensal de visita aos clientes, agregando os profissionais disponíveis, gerente e/ou os sócios. Assim, todo mês o cliente receberá pelo menos uma visita destes profissionais, para poder ter uma maior atenção voltada a ele, ajudando a resolver possíveis problemas.

Na dimensão Presteza, também foi identificado um alto nível de satisfação, apesar de alguns relatos destacarem a demora em fornecer informações e respostas pelos meios de comunicação. Não há nessa categoria necessidade de ação corretiva, mas sim preventiva para que se possa analisar o que está acontecendo que levou a tal insatisfação.

E por último, na dimensão Tangibilidade, houve um grande índice de neutralidade em relação às instalações físicas da empresa, dos equipamentos utilizados, da aparência dos profissionais e do sistema operacional utilizado. Sendo assim, concluiu-se que essa categoria pode ser irrelevante para os respondentes.

\section{Considerações finais}

Com este trabalho foi possível apurar que oferecer um serviço de qualidade, diante de um mercado cada vez mais exigente devido à competitividade, é um desafio para as empresas.

Diante do exposto, o objetivo deste artigo foi de analisar a qualidade do serviço prestado por um escritório de contabilidade na cidade de Franca, no estado de São Paulo, através da aplicação 
da survey, considerando o questionário baseado no modelo SERVPERF. Cada questão pôde ser avaliada de acordo com a priorização que deve ser dada para realizar melhorias, a fim de alcançar a satisfação de quem utiliza o serviço. Essas informações ajudam na gestão do escritório, fornecendo base para traçar estratégias de planejamento que visam à maximização da satisfação dos clientes.

Ademais, em relação aos resultados obtidos, o estudo mostra os pontos fracos e fortes do escritório contábil. Pode-se destacar como pontos fortes a satisfação dos clientes em diversos atributos. Como ponto fraco, observou-se um gap expressivo de qualidade no atributo sobre a empresa visitar os clientes em frequência suficiente.

Dessa maneira, com relação às contribuições para a prática, esse estudo ao experimentar a escala SERVPERF, contribuiu com resultados importantes ao proporcionar à empresa avaliada um feedback sobre o grau de serviço constatado pelos clientes, do mesmo modo que identificou possíveis clientes menos satisfeitos, o que possibilitará à empresa implantar estratégias específicas em cada caso, podendo assim recuperar os resultados positivos.

A pesquisa passou por algumas limitações, como a utilização de amostragem por conveniência e a falta de interesse dos clientes em responder o questionário, sendo utilizada uma amostra pequena para apuração dos resultados. Além disso, alguns clientes não possuíam e-mail, limitando assim a quantidade para o envio do questionário. E em relação ao modelo empregado, outras variáveis que não foram acrescentadas também podem ter interferência nas relações exploradas.

E por fim, para estudos futuros, sugere-se incluir alguns aspectos como a condução de um estudo longitudinal que avalie as variações nas características das compreensões indicadas pelos respondentes e também um estudo que realize uma avaliação qualitativa das opiniões das empresas que foram clientes do escritório para entender o motivo da transferência. Sugere-se também a aplicação de pesquisa semelhante a este presente estudo no ramo de atividade de escritórios contábeis, pois encontramos poucas referências nessa área.

\section{REFERÊNCIAS}

ANDRETTI, F. Análise do processo de entrega de serviços com base em modelo de correlação entre percepção e indicadores do processo. 2006. Dissertação (Mestrado Engenharia Industrial). PUC, Rio de Janeiro, 2006.

ASSIS, W. A. O papel da qualidade percebida na lealdade dos clientes de serviços de consultoria e assessoria prestados por empresas contábeis. 2013. 75 f. Dissertação (Mestrado em Controladoria Empresarial) - Universidade Prebisteriana Mackenzie, São Paulo, 2013. 
CARRILLAT, F. A.; et al. The validity of the SERVQUAL and SERVPERF scales: A metaanalytic view of 17 years of research across Five continents. Emerald Group Publishing Limited, 2007.

CERVO, A. L.; BERVIAN, P. A.; SILVA, R. Metodologia científica. 6 ed. São Paulo: Pearson Prentice Hall, 2007.

COELHO, C. D. A. Avaliação da qualidade percebida em serviços: aplicação em um colégio privado de ensino fundamental e médio. 2004. 179 f. Dissertação (Mestrado em Engenharia de Produção) - Universidade Federal de Santa Catarina, Florianópolis, 2004.

CONSELHO FEDERAL DE CONTABILIDADE. Resoluções. Brasília, 2018. Disponível em: 〈https://cfc.org.br/>. Acesso em: 27 jul. 2018.

CORRÊA, H. L.; CAON, M. Gestão de serviços: lucratividade por meio de operações e de satisfação dos clientes. São Paulo: Atlas, 2002.

CRONIN, J.; TAYLOR, S. Measuring service quality: A reexamination and extension. Journal of Marketing, v. 56, n. 3, p. 55-68, 1992.

FISCHER, G. et al. Gestão da qualidade: segurança do trabalho e gestão ambiental. 2. ed. São Paulo: Blucher, 2009.

GONÇALVES, T. J. M.; BELDERRAIN, M. C. N. Avaliação da qualidade em lan houses através da adaptação do instrumento SERVQUAL. Revista Produção Online, Florianópolis, SC, v. 12, n. 1, p. 248-268. 2012. Disponível em: <

https://producaoonline.org.br/rpo/article/view/882>. Acesso em 05 jul. 2018.

GRONROOS, C. Marketing, Gerenciamento e Serviços: a competição por serviços na hora da verdade. Rio de Janeiro: Campos, 1995.

HAIR JUNIOR., J. F. et al. Análise multivariada de dados. Porto Alegre: Bookman, 2005.

LOVELOCK, C. H.; et al. Marketing de Serviços: pessoas, tecnologias e estratégia. 7. ed. São Paulo: Pearson Prentice Hall, 2011.

LOVELOCK, C. H.; WRIGHT, L. Serviços: marketing e gestão. Rio de Janeiro: Saraiva, 2001.

MARTINS, R. A. et al. Guia para elaboração de monografia e TCC em engenharia de produção. São Paulo: Atlas, 2014.

OLIVEIRA, D. P. R. Manual de consultoria empresarial: conceitos, metodologia, práticas. 11. ed. São Paulo: Atlas, 2012.

OLIVEIRA, O. J. et al. Gestão da Qualidade: tópicos avançados. São Paulo: Pioneira Thomson, 2006.

OLIVER, R.L. A Cognitive model of the antecedents and consequences of satisfaction decisions, Journal of Marketing Research, v. 17, n. 4, p. 460-69, 1980.

PARASURAMAN, A; et al. A conceptual model of services quality and its implication for future research. Journal of Marketing, v. 49, n. 4, p. 41-50, 1985.

PARASURAMAN, A; et al. SERVQUAL: a multiple-item scale for measuring consumer perceptions of service quality. Journal of Retailing, v. 64, n. 1, p. 12 - 40, 1988.

PQEC. Cinco compromissos que a empresa participante do PQEC deve atender durante sua permanência no programa. São Paulo, 2005. Disponível em:

<https://www.pqec.org.br/>. Acesso em: 05 jul. 2018. 
TOLEDO, J. C.; et al. Qualidade: gestão e métodos. Rio de Janeiro: LTC, 2014.

YIN, R. K. Estudo de caso: planejamento e métodos. 5. ed. Porto Alegre: Bookman, 2015. 